\title{
Periocular Biometric Recognition Using Supervised Fuzzy Clustering
}

\author{
Vivek Srivastava, Bipin K. Tripathi, and Vinay K. Pathak
}

\begin{abstract}
Abtsract-Developing a potential biometrics has been a key focus of research in recent years. Periocular biometrics is a new trait to deal with non-ideal scenarios in face and iris biometrics. It can be used as an alternative to iris recognition, if the iris images are captured at a distance. In forensic applications, this trait can be used individually as well as with other traits (face and iris) for effective and accurate identification. In recent researches, the periocular biometrics is significantly impacting the iris and face based recognition. In this paper, we investigated the efficacy of supervised fuzzy clustering for strict periocular region which does not involve the eyebrows. The fixed initialization is considered in proposed supervised fuzzy clustering instead of random initialization. Then fuzzy clustering motivated with partition index maximization is used to optimize the objective function, hence yield clusters with representative prototype. The fuzzy clustering is further generalized with Minkowski distance matrices to yield variable cluster shape. Recognition is done based on the minimum distance measure between the test patterns and the centroid of the clusters. We use eight hundred periocular region images extracted from AR face dataset of $\mathbf{4 0}$ subjects. Performance of the proposed technique has been evaluated in terms of rank-one and rank- two recognition accuracy. Experimental analysis demonstrates the efficacy of presented technique over other variants of fuzzy clustering techniques.
\end{abstract}

Index Terms-Periocular biometrics, fuzzy clustering, supervised initialization, principal component analysis.

\section{INTRODUCTION}

The periocular biometric is gaining attention as a potential feature for biometric authentication in the recent past. The term periocular refers to the facial region in the immediate vicinity of the eye. It has been evolved as a separate modality in biometric information [1]-[3] which can be independently used for recognition. It has been also observed that it may aid to traditional biometric traits viz face, iris or retina, to identify an individual uniquely and reliably. The acquizistion of the periocular biometric requires less subject cooperation, where as it allows a larger depth of field compared to conventional ocular biometric traits. This trait can be more useful in forensic applications to reduce the search space when only periocular region is provided. One the influential factor for using this region as a separate trait is its non mandatory nature towards the higher resolution as compared

Manuscript received April 9, 2013; revised July 25, 2013

Vivek Srivastava is with the National Institute of Standards and Technology, Boulder, CO 80305 USA (e-mail: author@ boulder.nist.gov).

Bipin K. Tripathi was with Rice University, Houston, TX 77005 USA. He is now with the Department of Physics, Colorado State University, Fort Collins, CO 80523 USA (e-mail: author@lamar.colostate.edu).

Vinay K. Pathak is with the Electrical Engineering Department, University of Colorado, Boulder, CO 80309 USA, on leave from the National Research Institute for Metals, Tsukuba, Japan (e-mail: author@nrim.go.jp). to other ocular biometrics such as iris, retina and conjunctiva [4]-[6]. The fundamental requirement of any biometric recognition system is a human trait having several desirable features like universality, distinctiveness, permanence and acceptability. However, a human characteristic possessing all these features has not yet been identified. As a result, none of the single biometric trait can provide perfect recognition. It is also difficult to achieve very high recognition rates using single trait due to problems like noisy sensor data and non-universality or lack of distinctiveness of the chosen biometric trait. Therefore, the performance of a biometric system can be improved by utilizing a number of different biometric identifiers like face, iris, periocular, eye movement, etc. The result of combined effects will be more robust to noise and minimize the problem of non-universality and lack of distinctiveness. Due to the popularity of the face biometric, facial images have been extensively used for recognition. In this paper, we explore the utility of appearance based periocular features for biometric classification and present the feasibility of using the periocular region as a biometric trait. The work presented in this paper will be highly beneficial, when fusion of periocular biometrics will be done with the face. Recently, some of the techniques are developed for improving the recognition performance of biometric systems [4]-[6] using periocular features. They employ Local binary pattern [7]-[8], SIFT [3] and principal component analysis for feature extraction and different distance matrices techniques for classification. Few other computational intelligence based techniques including fuzzy clustering has proven their strength and efficacy for various kinds of classification problems [9]-[11]. Here, main rationale of introducing fuzzy clustering for biometric application is its high classification capacity especially under the overlapping classes. In most of the cases, fuzzy c-means clustering (FCM) with random initialization is used. The membership function and prototype matrix is iteratively updated based on an objective function [12]. In FCM, initialization plays a crucial role in generating the partition among the classes. Different initialization leads to different partitioning. Therefore, overcoming from this problem, variety of evolutionary algorithms are adopted [12]-[13]. Some of these algorithms also suffer with poor performance because of little compactness of classes and its unsupervised nature [12].

In order to avoid this nature, we propose a novel fuzzy clustering technique which adopts supervised initialization according to a priori known distribution of the classes. The supervised fuzzy clustering is done through assignment of each training object to its respective cluster with highest membership grade. The clusters with representative prototype are achieved by partition index maximization (PIM) methodology [14] which optimizes the objective function. Each cluster is represented by a prototype, which is the 
centroid of the members resides in a cluster for training set. For testing, we compute the distance between the test pattern and prototype of each class using a dissimilarity measure function based on Minkowski distance matrices. It is observed that this method outperforms over other existing variants of fuzzy clustering. The main motivation of this paper is to investigate the applicability of this novel clustering technique for strict periocular region recognition, the region which excludes the eyebrows.

The aim of paper is to explore whether periocular images carry enough information to reliably obtain similar biometric information to that obtained from face images. Of course, this biometric trait can be exploited with complete face to the improve recognition results of the biometric system. Rest of the paper is organized as follows- section 2 presents the feature extraction and novel fuzzy clustering with supervised initialization. Section 3 shows experimental results. The performance of the proposed system is obtained in terms of rank-one and rank-two accuracies. Finally section 4 is conclusion.

\section{Methodology}

As is shown in Fig. 1, a periocular region is the area around the eye which may be used separately to identify individuals. In recent papers, the periocular region which includes eyebrows is considered for recognition. The present work is intended to investigate the strength of periocular region without eyebrows, the region in strict environment. We use principal component analysis (PCA) for dimensionality reduction and dominant feature extraction [15]. In this section we formally develop a supervised fuzzy clustering technique for efficient periocular region recognition. Proposed fuzzy clustering involves the novel objective function inspired by PIM [14] with Minkowski distance. This method can be more suitable for forensic applications in reduced search space for identifying suspects, when periocular region is provided.

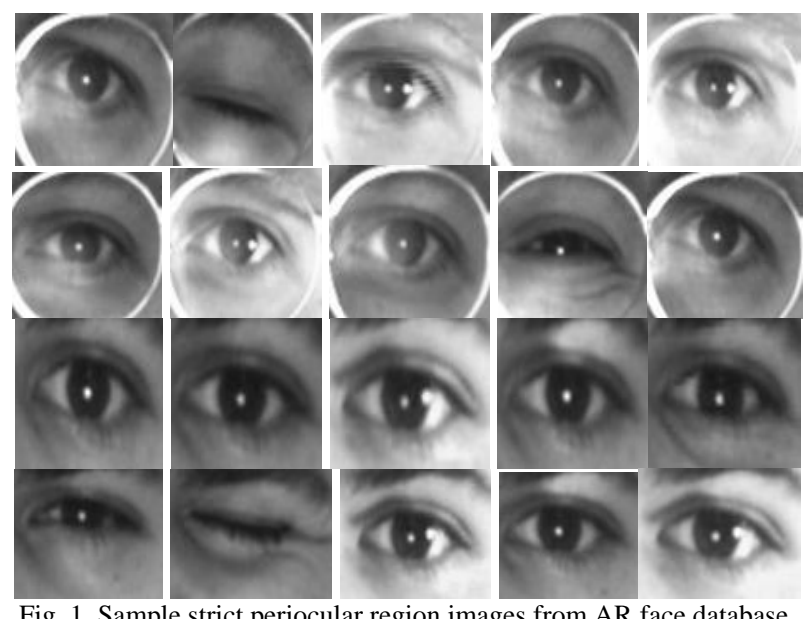

\section{A. Feature Extraction}

Principal component analysis is widely applied for dimensionality reduction and feature extraction based on extracting the preferred number of principal components of multivariate data [16]. In this statistical approach, the features are extracted from the whole image; they also maintain sufficient information for meaningful training. In experiments we observed that considering 40 principal components of training sets yield best results. Fig. 2 presents the eigen eyes corresponding to 05 highest eigen values extracted using PCA.
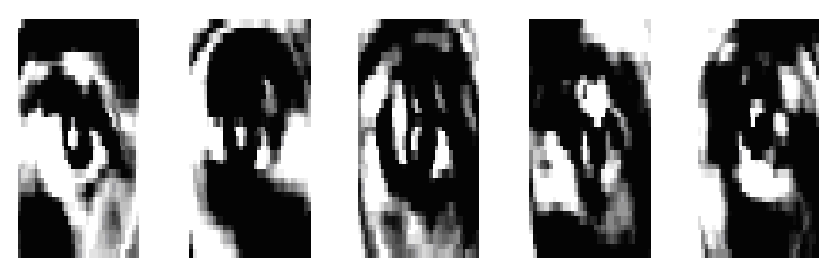

Fig. 2. The dominant eigen images for periocular region associated with highest 05 eigen values.

\section{B. Fuzzy Clustering with Supervised Initialization}

Let $\mathrm{X}=\left\{x_{1}, x_{2}, x_{3}, \ldots \ldots \ldots x_{N}\right\}$ is input dataset of considered images. Fuzzy clustering algorithm divides $\mathrm{N}$ datasets into fuzzy partition matrix $\mathrm{U}$ ( size $C \times N$ ) containing $C$ clusters. Membership function in $U$ is defined as $\mu_{i k}$ which satisfied the following constraints -

$$
\mu_{i k} \in[0,1] ; 0<\sum_{k=1}^{N} \mu_{i k}<N ; 0<\sum_{i=1}^{C} \mu_{i k}=1
$$

where, $\forall 1<i<C ; \quad 1<k<N ; \quad 2 \leq C \leq N$.

The objective function, we use here is motivated by a function described in PIM algorithm [14] for optimization. We further generalized it with Minkowski distance metrics. The Objective function which is defined over $\mu$ (membership grade) and $O$ (center of cluster) is defined as following:

$$
\begin{aligned}
& J(\mu, O)=\sum_{k=1}^{N} \sum_{i=1}^{C}\left(\mu_{i k}\right)^{m} d^{2}\left(x_{k}, O_{i}\right)-w \sum_{k=1}^{N} \sum_{i=1}^{C}\left(\mu_{i k}\right)^{m} \\
& w>0 \\
& d\left(x_{k}, O_{i}\right)=\left(\sum_{j=1}^{n}\left\|x_{k j}-O_{i j}\right\|^{p}\right)^{1 / p}, 1 \leq p<\infty,
\end{aligned}
$$

where, $m$ is a weighting exponent which is known as fuzzifier. Generally, the value of $m$ lies between one to infinity. Value of $\mathrm{m}$ greatly influences the performance of fuzzy clustering. Therefore selection of suitable fuzzifier ' $m$ ' is very important for implementation of fuzzy clustering. In most of the cases value of $\mathrm{m}$ is selected based on the dataset/problem, as there is no rigorous criterion for selection of this parameter. Parameter $w$ is same as in partition index maximization (PIM) algorithm [16]. $d\left(x_{k}, O_{i}\right)$ is Minkowski distance taken in objective function. Main motivation behind using Minkowski distance in stead of Euclidian distance is to provide more generalization with a parameter $p$. The nature/value of this parameter decides the shape of clusters to be generated, which may be boxes, ellipsoids, spheres and others. Selection of this distance measure does not tend the 
shape of cluster spherical which is often in Euclidian distance. The updates in centre of the clusters and member function are defined as:

$$
\begin{gathered}
\mathrm{O}_{i}=\frac{\sum_{k=1}^{N} \mu_{i k}{ }^{m} x_{k}}{\sum_{k=1}^{N} \mu_{i k}{ }^{m}} \\
\mu_{i k}=\frac{\left(d\left(x_{k}, O_{i}\right)-w\right)^{\frac{1}{\beta(m-1)}}}{\sum_{j=1}^{C}\left(d\left(x_{k}, O_{i}\right)-w\right)^{\frac{1}{\beta(m-1)}}}, i=1,2 \ldots ., C
\end{gathered}
$$

The membership update function used in [14] is further modified with $\beta$ (Eq. 4) where $0<\beta<1$ to evolve better partitioning. This parameter provides proper allocation of members into clusters in training phase. Let $J(\mu, O)^{(t)}$ is the objective function at $\mathrm{t}$ th iteration then The algorithm terminate with the condition --

$$
\left\|J(\mu, O)^{(t+1)}-J(\mu, O)^{(t)}\right\|<\mathfrak{I},
$$

where $\mathfrak{J}$ is the pre specified threshold.

\section{Training}

The considered eye image training dataset (strict periocular region) possesses 400 images. We have use 200 images, containing 5 images per persons, for training. Training process involves the fuzzy clustering with supervised initialization which distributes these 200 images into 40 clusters. In the supervised initialization, first five images are assigned to first cluster, next six to ten images are assigned to second cluster and so on with membership grade 0.9, as shown in Table I. As the above algorithm runs, we get new distribution of images into 40 clusters which are finally used in testing phase.

\begin{tabular}{|l|l|l|l|l|l|l|l|l|}
\hline & 1 & -- & 5 & 6 & -- & 10 & -- & 200 \\
\hline C1 & 0.9 & 0.9 & 0.9 & .002 & .002 & .002 & --- & 0.002 \\
\hline C2 & .002 & -- & -- & 0.9 & 0.9 & 0.9 & --- & .002 \\
\hline$:$ & --- & ---- & -- & --- & -- & -- & -- & -- \\
\hline C40 & .002 & --- & --- & ---- & -- & --- & 0.9 & 0.9 \\
\hline
\end{tabular}

\section{Testing}

In this phase, we use following distance measure which is a Minkowski distance, having a generalization parameter ' $p$ ' defined as

$$
d\left(\text { test }_{k}, O_{i}\right)=\left(\sum_{j=1}^{n}\left\|\operatorname{test}_{k j}-O_{i j}\right\|^{p}\right)^{1 / p}
$$

' $d$ ' calculates the distance between input test pattern and the prototype of the cluster. Since we are using Minkowski distance measure in objective function which is used in training phase, therefore it has been observed that we get best results at $p=4$ instead of using $p=2$ which is often used. Test pattern having least distance with $O_{i}$ is recognized for corresponding cluster $C_{i}$. Testing is done on different set of data which also contains 200 images for both left and right eye periocular region.

\section{EXPERIMENTAL RESULTS}

In order to evaluate the proposed methodology, we use eye dataset extracted from AR face database [17]. AR face dataset contains variation in images in terms of occlusion, expression and illumination. All images from session 1 are used for selection of periocular region except images with sun glasses. As is shown in Fig. 3, we have selected 800 images from 400 images of faces in which 2 periocular regions are extracted from single image for left and right eyes respectively. Each experiment is performed on single periocular region. Final accuracy is obtained by combining the results of left and right eyes.

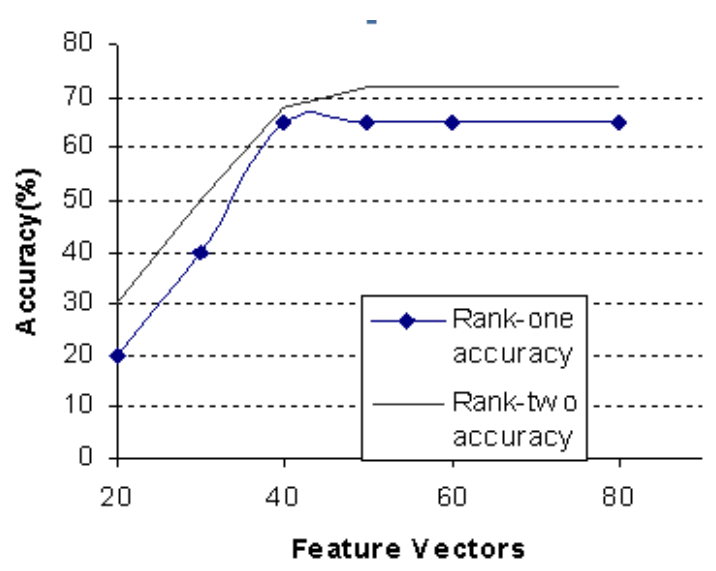

Fig. 3. Plot between accuracy and number of feature vector selected.

\section{A. Performance Evaluation}

TABLE II: COMPARATIVE ANALYSES AMONG EXISTING TECHNIQUES

\begin{tabular}{|l|l|l|l|}
\hline & $\begin{array}{l}\text { Objective } \\
\text { Function }\end{array}$ & $\begin{array}{l}\text { Rank-one } \\
\text { Accuracy }\end{array}$ & $\begin{array}{l}\text { Rank- Two } \\
\text { Accuracy }\end{array}$ \\
\hline FCM & 6274.3 & $12 \%$ & $21 \%$ \\
\hline EFC-MD & 6211.6 & $51 \%$ & $59 \%$ \\
\hline PIM & 6273.2 & $40 \%$ & $51 \%$ \\
\hline PIM-SI & 6317.5 & $50 \%$ & $61 \%$ \\
\hline $\begin{array}{l}\text { Proposed } \\
\text { method }\end{array}$ & 6148.4 & $65 \%$ & $72 \%$ \\
\hline
\end{tabular}

To evaluate the performance of the proposed technique, Rank-one and rank- two recognition accuracy is used. Rank-one accuracy is defined as the ratio between the correctly matched patterns and total input patterns. Rank-two accuracy means that correct classification is recorded when a correct match for testing image is one of the two nearest images in subspace. We have analyzed the performance of the proposed technique with standard FCM, evolutionary fuzzy clustering with Minkowski distances (EFC-MD) [18], 
Partition index maximization (PIM) algorithm [14], PIM with supervised initialization (PIM-SI). It has been observed from the Table II that proposed technique outperforms over other clustering techniques. Present method gets $65 \%$ as compared with maximum accuracy of 62.59 obtained in [1] without considering eyebrows. This method also takes lesser number of features as compared with LBP technique because we use PCA for feature extraction. Table III shows the results when we are selecting left eye periocular region for training and testing is performed for both left and right eye periocular regions. It is clear that we get maximum accuracy (rank-one) of $66 \%$ when same eye regions are selected for training as well as testing.

TABLE III: Results OF PROPOSED TECHNIQUe ON SELECTING DiFFERENT TRAINING/TESTING SET

\begin{tabular}{|c|c|c|c|}
\hline & $\begin{array}{c}\text { Left train } \\
\text { (Training Set) }\end{array}$ & $\begin{array}{c}\text { Left Test } \\
\text { (Testing Set) }\end{array}$ & $\begin{array}{c}\text { Right Test } \\
\text { (Testing Set) }\end{array}$ \\
\hline Accuracy & $54.5 \%$ & $66 \%$ & $64 \%$ \\
\hline
\end{tabular}

\section{B. Effect of Illumination}

In the dataset used, three images are affected with illumination factor out of ten images per person. For this evaluation, we have separated these 3 images of each subject. Hence total 280 images for each eye are considered. Out of 280 images for each eye, 140 images are used for training and 280 images are used for testing. Accuracy is increased by $2 \%$ on excluding these images.

\section{CONCLUSION}

We have investigated the efficacy of proposed fuzzy clustering using periocular biometrics. This method can be more suitable for forensic applications in reduced search space for identifying suspects, when strict periocular region (excluding eyebrows) is provided. The proposed fuzzy clustering uses a novel objective function with considering Minkowski distance and supervised initialization. We have also introduced a parameter $\beta$ in membership updating function which provides better partitioning. Rank-one recognition accuracy for this periocular images without eyebrows extracted from AR face dataset was $65 \%$ which clearly indicates the efficacy of proposed technique over other existing variants of fuzzy clustering techniques.

\section{REFERENCES}

[1] U. Park, A. Ross, and A. K. Jain, "Periocular biometrics in the visible spectrum: a feasibility study", in Proc. the 3rd IEEE International Conf. on Biometrics: Theory, Applications and systems, 2009, pp. $153-158$.

[2] S. Bharadwaj, H. S. Bhatt, M. Vatsa, and R. Singh, "Periocular biometrcs when iris recognition fails," in Proc. 4th IEEE Conf. on BTAS, 2010, pp. 1-6.

[3] U. Park, R. Jillala, A. Ross, and A. K. Jain, "Periocular biometrics in visible spectrum," IEEE Trans on Information Forensic and Security, vol. 6, no. 1, pp. 96-106, 2011.

[4] J. Adams, D. L. Woodard, G. Dozier, P. Miller, K. Bryant, and G. Glenn, "Genetic-based type II feature extraction for periocular biometric recognition: less is more," in Proc. 20th International Conf. on Pattern Recognition, 2010, pp. 205-208.
[5] J. R. Lyle, P. E. Miller, S. J. Pundlik, and D. L. Woodard, "Soft biometric classification using periocular region features," in Proc. 4th IEEE Conf. on BTAS, 2010, pp. 1-7.

[6] J. Merkow, B. Jou, M. Savvides, "An exploration of gender identification using only the periocular region," in Proc. 4th IEEE Conf. on BTAS, 2010, pp 1-5.

[7] T. Ahonen, A. Hadid, and M. Pietikainen, "Face description with localbinary patterns: Application to face recognition," IEEE Trans. on Pattern Analysis and Machine Intelligence, vol. 28, no. 12, pp. 2037-2041, 2006

[8] T. Ojala, M. Pietikainen, and T. Maenpaa, "Multiresolution gray-scale and rotation invariant texture classification with local binary patterns," IEEE Trans. on Pattern Analysis and Machine Intelligence, vol. 24, no. 7, pp. 971-987, 2002.

[9] A. alford, K. Popplewell, G. Dozier, K. Bryant, J. Kelly, J. Adams, T. Abagaz, J. Shelton, K. Ricanek, and D. Woodward, "A Comparison of GEC-Based Feature Selection and Weighting for Multimodal Biometric Recognition," IEEE Congress on Evolutionary Computation, pp. 2725-2728, 2011.

[10] P. Fazendeiro and J. V. D. Oliveira, "A semantic driven evolutive fuzzy clustering algorithm," in Proc. IEEE Int Conf. Fuzzy Syst., 2007, pp. 1-6.

[11] X. W. Yang, G. Zhang, J. Lu, and J. Ma, "A kernel fuzzy c-means clustering-based fuzzy support vector machine algorithm for classification problems with outliers or noises," IEEE Trans on Neural Network, vol. 19, no. 1, pp. 105-115, February 2011.

[12] E. R. Hruschka, R. J. G. B. Campello, A. A. Freitas, A. C. P. L. F. D. Carvelho," A survey of evolutionary algorithms for clustering," IEEE Trans. on System Man and Cybernetics-Part C:Applications and Reviews, vol. 39, no. 2, pp.133-155, 2009.

[13] K. Deb, Multi-Objective Optimization Using Evolutionary Algorithms, New york: Wiley, 2001.

[14] G. L. Marcialis and F. Roli, "Fusion of appearance based face recognition algorithms," Pattern Analysis and Applications, vol. 7, no. 2, pp 151-163, July 2004.

[15] M. A. Turk and A. P. Pentland, "Eigenfaces for recognition," $J$ Cognitive Neurosci., vol. 3, pp. 71-86, 1991.

[16] D. Ozdemir and L. Akarun, "A fuzzy algorithm for color quantization of images", Pattern Recognition, vol. 35, pp. 1785-1791, 2002.

[17] R. Benavente et al., "The AR face database," CVC Technical Report, pp. 24, 1998.

[18] V. Srivastava, B. K. Tripathi, and V. K. Pathak, "An evolutionary fuzzy clustering with minkowski distances," in Proc. Intl. Conf. on Neural Information Processing (ICONIP), 2011, pp. 753-760.

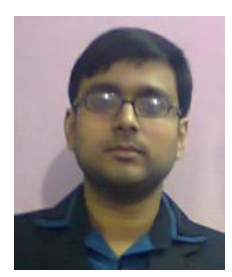

Vivek Srivastava is pursuing Ph.D. degree from Gautam Buddh Technical University, Lucknow. His research interest fields include computational intelligence, biometrics and data mining. He has published more than 8 papers in various international journals and conferences. Presently he is working as a teacher fellow in CSE department, HBTI, Kanpur. He is a member of IACSIT, IAENG and NCIRG.

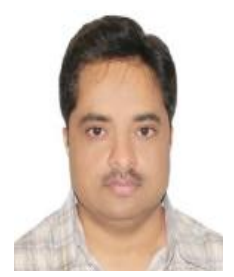

Bipin K. Tripathi received M.Tech. degree and Ph.D. degree from IIT Delhi and IIT Kanpur respectively. He is currently working as an associate professor at HBTI, Kanpur. His research interest fields are complex valued neural network, high dimensional neuron model and soft computing. He has published more than 25 publications in various international journals including IEEE transaction papers. He is also the founder member of nature-inspired computational intelligence research group (NCIRG).

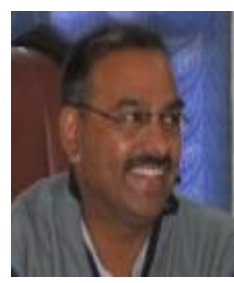

Vinay K. Pathak received M.Tech. and Ph.D degree from IIT Kharagpur and Uttar Pradesh technical university Lucknow respectively. He is currently working as a professor at HBTI, Kanpur. Earlier he worked as the vice chancellor of Uttarakhand open university, Haldwani. He has published more than 80 research publications in various international journals and conferences. His research fields are image processing, biometrics. He is also a member of various research groups, technical associations etc. 